\title{
FENOMENOLOGÍA, VERDAD Y HORIZONTE ${ }^{1}$
}

\author{
Daniel Michelow ${ }^{2}$
}

RESUMEN: El presente articulo retoma la larga discusión sobre la relación entre los principales conceptos de verdad de la fenomenología - la evidencia (Evidenz) husserliana y el estar al descubierto (Entdecktheit) heideggereano - para argumentar contra las dos vertientes analíticas imperantes que, transitando caminos diferentes, han arrojado sin embargo la misma conclusión en cuanto a una insalvable mutua exclusión entre tales conceptos. A continuación se darán algunas claves que permitirán exponer tales conceptos como modos complementarios de un fenómeno de verdad mas amplio, para finalmente y en base a esta exposición poner de relieve de forma critica ciertos aspectos de la comprensión de historia de Heidegger que como se mostrará han repercutido negativamente en una visión unificadora de la fenomenología.

Palabras clave: Evidencia. Estar al descubierto. Verdad. Historia. Fenomenología.

Principalmente son dos las lecturas que dificultan el intento por otorgarle a los principales conceptos de verdad de la fenomenología - "evidencia" (Evidenz) en Husserl y "estar al descubierto"3 (Entdecktheit) en Heidegger una cierta coherencia y unidad. La primera lectura, que surge tras la segunda guerra mundial, guarda relación con una supuesta deficiencia del concepto de "estar al descubierto" para asegurarle al conocimiento nociones normativas. La segunda lectura, que guarda relación con una supuesta independencia total del concepto de verdad de Heidegger de la tradición que le precede, surge aparentemente, desde la obra misma de este.

La primera lectura se origina con la aparición de Der Wabrheitsbegriff bei Husserl und Heidegger ${ }^{4}$ de Ernst Tugendhat el año 1967 que marca un

\footnotetext{
${ }^{1}$ http://dx.doi.org/10.1590/S0101-31732018000100010

2 Pontificia Universidad Católica de Chile, Santiago, Chile. E-mail: daniel.michelow@gmail.com.

Doctor en filosofía, Heidelberg Universität. Actualmente postdoctorando en la Pontificia Universidad Católica de Chile. Este trabajo ha sido realizado en el marco del proyecto Fondecyt de Postdoctorado número 3180019 de CONICYT, Chile.

${ }^{3}$ Todas las citas de Heidegger refieren a la Gesamtausgabe mediante las siglas GA, seguidas del número de volumen y página. Para las citas provenientes de Ser y tiempo hemos optado por la versión castellana de E. Rivera (Ser y tiempo. Santiago: Editorial Universitaria, 2015) que incluye a su vez la original.

${ }^{4}$ Todas las traducciones de este texto para el presente análisis serán de autoría propia.
} 
hito en la investigación de estos autores en el sentido de que esta obra le abre camino a la tesis de que los conceptos respectivos de verdad se encontrarían - bajo la perspectiva de la así llamada "responsabilidad critica" (kritische Verantwortlichkeit) - en un conflicto insalvable. La perspectiva abierta por Tugendhat, que abala este modo especifico del conflicto entre tales conceptos, ha prevalecido hasta hace pocos años sin mayor contrapeso, eclipsando otras posibilidades de análisis ${ }^{5}$. Esta perspectiva en la que Tugendhat pone el pensamiento de Heidegger ha retomado fuerza especialmente tras la aparición de los llamados cuadernos negros (HEIDEGGER, GA 94-97).

La obra de Tugendhat lleva a cabo su análisis a la luz de lo que él comprende como un estar en "relación a la verdad" (Wabrheitsbezug), con lo que quiere dar a entender que al hecho inmediato y puro de la verdad le es necesario un momento de referencia subjetiva. Esta relación es para Tugendhat la condición necesaria para la concreción del fenómeno de la verdad. Dicha concreción es de antemano imposible de llevar a cabo desde un proyecto como el de Heidegger que conlleva - en las palabras del propio Tugendhat - un "[...] radical alejamiento de la filosofía de la subjetividad” (TUGENDHAT, 1984, p. 385), pues una vez perdido el referente subjetivo se volvería necesariamente imposible la instancia en que la vida humana asume una posición normativa y critica respecto de la verdad.

Es justamente desde este enfoque desde el cual Tugendhat asevera que en Husserl - a diferencia de su discípulo - "[...] el concepto de verdad tiene una posición central y con ello gana relevancia universal." (TUGENDHAT, 1984, p. 5). Tugendhat hace referencia a que el padre de la fenomenología a través de su concepto de evidencia se ha hecho cargo de la necesidad de construir una relación determinante desde el sujeto con aquello que se da sin más como verdadero, con lo que se respetaría la capacidad normativa de este como momento constitutivo de lo verdadero.

Esta descripción no solo muestra la posición filosófica de Tugendhat respecto del momento específico de la relación entre Husserl y Heidegger como portavoces de la fenomenología, sino que además deja entrever su comprensión general de los modos de operar del pensamiento filosófico, pues lo que subyace a tal análisis es que estos conceptos son intentos aislados de abordar un asunto idéntico y estático, con lo que ignora por completo la dimensión histórica del problema o mas bien la reduce a un mero asunto

\footnotetext{
${ }^{5}$ Entre otros reconocidos autores vale la pena nombrar aquí a Adorno (1970) y Habermas (1988), quienes en base al trabajo de Tugendhat han construido una lectura critica de Heidegger.
} 
historiográfico en el que no hay un contexto de co-relación entre la pregunta y lo preguntado. Es desde esta comprensión general del desarrollo de la filosofía, desde la cual se puede medir con una vara única ambos proyectos, pudiéndose hacer juicios respecto de la prevalencia del uno sobre el otro.

El resultado general de tal comprensión historiográfica de la tradición filosófica es que los diversos proyectos de pensamiento aparecen como confrontados a un objeto absoluto, negándose así sus diversos retos y necesidades propias respecto de un asunto en constante cambio, que requiere siempre modos distintos de acceso. Esto repercute específicamente en relación a la interpretación realizada por Tugendhat del pensar de Heidegger en que siendo juzgado este desde una comprensión que no reconoce modulaciones históricas, determinadas desde el asunto mismo a pensar, se dan por descartado de plano momentos de estar en relación a la verdad y su consecuente evaluación que no responden a patrones normativos. La comprensión ahistórica de Der Wahrheitsbegriff determina como única posibilidad de relacionarse evaluativamente a la verdad la evaluación critico-normativa. Pero como se vera a continuación, si bien toda norma es evaluativa, no toda evaluación es necesariamente normativa.

Algo que por otro lado si logra encontrar Tugendhat en Husserl, ya que en su fenomenología la evidencia, esta comprendida no solo como la "dación originaria" (originäre Gegebenheit) del objeto sino que además como la corroboración de una "síntesis de identificación" (Deckungssynthese) entre contenidos de conciencia surgidos en este darse. En la evidencia entendida como criterio de la correspondencia sintética, encuentra Tugendhat la posibilidad de la demandada relación normativa a la verdad.

Desde esta exposición es posible entender que en Der Wahrheitsbegriffse ignoren elementos claves en la descripción del estar al descubierto llevado a cabo por Heidegger en Ser y tiempo. Elementos que incluso a primera vista pueden ser catalogados justamente como modos de evaluación y sin duda alguna como señales de un modo de estar en relación a la verdad. Principalmente quiero hacer referencia aquí a lo que Heidegger llama "evidenciación" (Ausweisung) y que utiliza en el siguiente contexto:

Pero el enunciado que ha sido expresado es un ente a la mano, y lo es de tal manera que, en cuanto conserva en sí el estar al descubierto, tiene en sí mismo una relación con el ente descubierto. La evidenciación [Ausweisung] de su ser-descubridor significa ahora: evidenciación de la relación que 
tiene con el ente el enunciado que conserva el estar al descubierto. (HEIDEGGER, GA 2, p. 224).

En vistas de esta descripción se torna claro que no se está frente a un fenómeno de verdad inmediato como se ha pretendido, sino que a uno que requiere a su vez también de su propia y particular concreción. Decididamente se evita en Ser y tiempo la palabra "evidencia" con el fin de acentuar las diferencias programáticas con Husserl, esto tiene su sentido en que en Ser y tiempo se pretende un tipo de evaluación que no trasgreda el ámbito fenomenológico del aparecer mismo. Heidegger busca la evidenciación fenomenológica, es decir ya no mas un tipo de evaluación que enfrente al ente, mediante "[...] un acto propio de descubrimiento" (HEIDEGGER, GA 2, p. 222), sino que solo al modo de dejar aparecer el propio estar al descubierto de este.

Si bien no es posible profundizar aquí este tema, si es posible por lo pronto prestar atención a las primeras similitudes que se nos presentan entre los proyectos en cuestión, para, por lo menos someramente, restablecer un puente entre el fenómeno descrito en Ser y tiempo y lo propuesto por Husserl, en el sentido de que ambas fenomenologías fundan en fenómenos de la verdad estructurados en torno a un momento de aparición del ente mismo. Por lo pronto, parece ser cuestión necesaria para evitar la disgregación de los conceptos en cuestión evitar la línea interpretativa abierta por Tugendhat en la que la relación a la verdad puede darse exclusivamente como evaluación normativa y toma de posición critica. Como expresé con anterioridad, esta limitación en la descripción de Tugendhat viene dada por su comprensión ahistórica y estática del pensar en general que repercute en un punto ciego respecto de que el estar al descubierto implica necesariamente también una relación a la verdad, esta ahora en un contexto fenomenal y no normativo. Es decir, ya no mas poniendo de relieve la experiencia subjetiva que se hace del ente aparecido, sino que acentuando la vida humana (al Dasein) como condición de posibilidad para el aparecer mismo.

La segunda lectura ${ }^{6}$ a la que he hecho referencia en un comienzo se origina en parte en la filosofía de Heidegger mismo así como en un tipo de interpretación tendenciosa y meramente negativa de la relación de su pensamiento para con la tradición filosófica que le precede. Esta interpretación insinuada desde la constelación terminológica de su programa histórico-filosófico, por ejemplo desde el binomio destrucción (Destruktion) y superación (Überwindung), así

${ }^{6}$ Con matices y formas diversas se encuentra este tipo de lectura en autores como Herrmann (2002), Martel (2008), Gadamer (2010) y Sallis (2012) entre otros. 
como desde el uso que se le da a los términos "origen" (Ursprung) y "vuelta" (Kehre), por nombrar algunos, da cabida a una comprensión de la filosofía heideggereana como un pensamiento desligado y aislado de una tradición indiferenciada, que supeditada a un rasgo simplificador, puede ser reunida bajo un titulo único. El titulo es, como sabemos, "metafísica". Dentro de este grupo cae, por supuesto, también Husserl, ya que su fenomenología es - para la lectura en cuestión - una forma más de esta tradición.

Es por cierto un despropósito atribuir a Heidegger completamente este tipo de lectura, ya que el mismo matiza frecuentemente en un sentido general estos términos, aseverando que no están para desestimar la tradición (HEIDEGGER, GA 2, p. 22). Aún así es posible encontrar innumerables ejemplos prácticos de la implementación negativa de los mismos, por lo que aun cabe preguntarse si esta lectura se corresponde o no con el espíritu del proyecto fenomenológico de Heidegger. Importante es para la presente investigación, reparar en que de hecho, no es posible encontrar luces en su obra de que este tan siquiera sopese la posibilidad de que justamente el centro mismo de su proyecto, esto es el concepto de verdad, se deba de algún modo, ni pueda ser tan siquiera entendido como un complemento ${ }^{7}$ del concepto de evidencia.

Son ya conocidas las razones que esgrime Heidegger respecto de por que el fenómeno de verdad con el que él ha dado, "[...] no tiene, pues, en absoluto, la estructura de una concordancia entre conocer y objeto, en el sentido de una adecuación de un ente (sujeto) a otro (objeto).” (HEIDEGGER, GA 2, p. 217). Estas tienen que ver, como es sabido, especialmente con que el concepto de estado de descubierto no pretende instaurarse como un relato más en el que se de cuenta ante todo el modo en que el sujeto participa y determina, es decir, como se entiende a si mismo en la relación a la verdad. A este tipo de relaciones tradicionales se les puede llamar, en términos simples, relaciones comparadoras. En este sentido dice Heidegger que "[...] lo que necesita ser evidenciado es únicamente el estar-descubierto del ente mismo, de él en el cómo de su estar al descubierto." (HEIDEGGER, GA 2, p. 216). Como indique anteriormente no se descarta así que el Dasein este en una relación a la verdad, ni tampoco que esta relación sea de carácter evaluativo, sino que se la pretende reconducir a un ámbito puramente fenomenal. Esto lleva a Heidegger a aseverar que la verdad descubierta en su carácter original no puede por tanto guardar ningún

\footnotetext{
7 La complementación esta entendida acá como un fenómeno positivo, que no se limita a lo que Heidegger llama reconducción (Rückführung), que tiene mas bien un carácter reductivo, es decir con la intención de mostrar un fenómeno en su carácter derivado (HEIDEGGER, GA 2, p. 213).
} 
parentesco con las modulaciones que ha entregado la metafísica, pues estas siempre tienen su punto de partida en una mirada que se sirve de una relación de verdad para ejercitar una comparación. No importa cual sea la variante en cuestión, ni siquiera si una de estas, como la de Husserl, implica un darse originario. Las nuevas reglas del juego son claras y afectan a todo el conjunto postsocratico y por supuesto a la evidencia por igual:

No se comparan representaciones entre sí, ni tampoco en relación a la cosa real. Lo que ha de evidenciarse no es una concordancia del conocer y el objeto, ni menos aun de lo psíquico y lo físico, pero tampoco es una concordancia de "contenidos de conciencia" entre sí. (HEIDEGGER, GA 2, p. 216).

Hasta aquí la exposición del problema transita por un camino interpretativo conocido y en cierto modo uno al cual es difícil mostrarle cierta oposición, sobre todo si se considera que el propio Husserl define sin más la verdad como "[...] completa correspondencia entre lo mentado y lo dado" ${ }^{8}$ (HUSSERL, HUA 19, p. 651), y paso seguido a la evidencia como la vivencia de tal correspondencia. De este modo, de las tres criticas que desliza Heidegger al concepto de verdad tradicional en la frase anterior, a saber, a) en general el carácter comparativo y no fenomenal de la relación de verdad; derivadamente b) el problema que surge de la relación comparativa de ámbitos ontológicos distintos, y por ultimo c) el problema de la preeminencia teorética en la relación entre representaciones (o contenidos de conciencia, si se quiere), la primera y la tercera parecen a primera vista recaer con fuerza en Husserl.

A pesar de que en base a la relación que pretende establecer Heidegger con la tradición y a la definición otorgada por el mismo Husserl el tema debiera darse por zanjado, creo necesario aquí hacer mención a varios aspectos importantes en relación al concepto de este ultimo que den mas profundidad a la relación filosófica de ambos proyectos.

Por un lado es necesario notar que una lectura detallada de obras como La idea de la fenomenología y las Investigaciones lógicas arroja una cierta ambigüedad en la comprensión de la evidencia que tiende a rebasar la definición principal de verdad de Husserl. Esta ambigüedad no es

\footnotetext{
8 Todas las citas de Husserl refieren a la Husserliana mediante las siglas HUA, seguidas del numero de volumen y página. Para las citas provenientes de las Investigaciones lógicas hemos optado por la traducción castellana de M. García Morente y J. Gaos (Investigaciones lógicas. Madrid: Alianza Editorial, 1999) que incluye a su vez la paginación original.
} 
aquella entre una verdad lógica y una verdad fenomenológica como ya se ha investigado ${ }^{9}$ profusamente, sino que una circunscrita netamente dentro del ámbito fenomenológico. Este tema ya ha sido tratado en los últimos ańos ${ }^{10}$, pero las conclusiones, por diversas razones ${ }^{11}$, no han sido hasta ahora dispuestas en la dirección de una cierta unificación del núcleo de la fenomenología como aquí se pretende.

La ambigüedad a la que se hace referencia es la que someramente se ha insinuado en un principio al definir el concepto de evidencia tanto como síntesis de identificación así como dación originaria. No se trata aquí de aseverar que Husserl no haya estado consciente de tal diversidad de significados, sino que mas bien de que la dación en el modo de estructuración del concepto de verdad husserliano ha quedado supeditada al momento sintético. La sintesis hace uso de la dación originaria solamente para rebasar el limite de la verdad proposicional, $y$ asi poder refundar el ámbito cientifico de conocimiento, pero no para superar la estructura de concordancia.

A pesar de que Husserl, en pos de su programa, acentúa el aspecto sintético de la evidencia, haciéndose blanco de la critica heideggereana, es necesario hacer notar - a pesar de que parezca obvio - que la capacidad de la conciencia de sintetizar sus contenidos, es solo posible gracias al momento de la dación originaria, que, tematizado o no, abre un ámbito de posibilidades puramente fenomenal, que no puede ser descartado como un mero modo de relaciones comparativas.

Si bien las señales que avalan este tipo de lectura se muestran solo tímidamente, están presentes en toda la obra de Husserl, pues lo que ocurre en la dación efectivamente no trata ni de la generación de contenidos, ni de una pretensión de poner de relieve las relaciones entre tales contenidos representacionales, sino mas bien el hecho simple y original de que el objeto se da y como se da. El propio Husserl da cuenta en el siguiente pasaje de una multiplicidad de sentidos del concepto de evidencia:

\footnotetext{
9 Respecto de este tema se pueden consultar por ejemplo Lugo (1970), Heffernan (1983) y Herrera (2011).

10 Principalmente Zahavi (2009).

${ }^{11}$ Zahavi propone en base a la ambigüedad detectada en la comprensión de verdad de Husserl reconsiderar la relación - unidad y diferencia - entre evidencia y estar al descubierto respecto de la presencia o ausencia en tales conceptos de disposición hacia el conocimiento. Este modo de análisis parece no ser fructífero en cuanto a que la caracterización del estar al descubierto como un fenómeno que no guarda relación con el conocer hace recaer el análisis en la escisión planteada por Tugendhat entre una verdad pura, del ser y otra distinta, del ente.
} 
[... la reducción fenomenológica no significa la limitación de la investigación a la esfera de la inmanencia ingrediente, a la esfera de lo incluido como ingrediente en el „esto que está aquí “ absoluto de la cogitatio; no significa en modo alguno la limitación a la esfera de la cogitatio, sino la restricción a la esfera de lo que se da puramente en sí mismo; a la esfera de aquello de que no sólo se habla y que no sólo se mienta - tampoco a la de lo que se percibe -; sino a la esfera de lo que está dado exactamente en el sentido en que está mentado, y dado ello mismo en el más estricto sentido, de tal modo que nada de lo mentado deja de estar dado. En una palabra: restricción a la esfera de la pura evidencia - entendido este término en cierto estricto sentido que excluye ya la „evidencia mediata" y, sobre todo, toda evidencia en sentido laxo. (HUSSERL, HUA II, p. 60-61).

En base a este y otros pasajes respecto de los diversos modos de la evidencia es posible hacer frente a otra de las criticas de Heidegger dirigida contra Husserl en relación a que el "Dasein no necesita colocarse ante el ente mismo en una experiencia 'originaria', $y$, sin embargo, su ser queda vuelto hacia ese ente.” (HEIDEGGER, GA 2, p. 222). Esto a diferencia de la conciencia intencional, según se quiere dar a entender. Sin embargo es posible aseverar ahora, que la conciencia tampoco necesita colocarse ante el ente mismo para obtener evidencia de un estado de cosas dado - no por lo menos cuando nos mantengamos dentro de "la esfera de la pura evidencia" y no en el ámbito de la evidencia mediata, que requiere efectivamente estar ante el ente "en persona" (leibhaft) o la evidencia en sentido laxo, que, como se ha dicho, refiere a la consistencia de una síntesis. La evidencia pura no esta referida ya al ente dado en la percepción (Wahrnehmung), sino que al ente dado fenomenalmente en la intuición (Anschauung) y que permanece de esta forma en la conciencia. La evidencia pura es entonces la reconducción de la conciencia a tal dación que se instaura como el modo fenomenal de acceso al ente mismo.

Lo que Heidegger ataca es especialmente el poner de relieve las relaciones de concordancia dentro de la esfera de la conciencia que sin duda se dan en la evidencia como síntesis, pero no acá. La dación originaria es en términos del pensar husserliano, el hecho transcendental per se. Incluso de modo aun mas decidido que la "cosa real", que tiene para Husserl el mero status de información sensual, pues el lugar de su persistencia es la conciencia, sin originarse en esta de ningún modo, es decir, sin implicar ningún grado de determinación inmanente. 
Las Investigaciones lógicas pueden ser entendidas como una obra guiada por la pregunta general por la condición de posibilidad de como los objetos transcienden en la conciencia. Este preguntar general no trata de esclarecer como la conciencia administra tales objetos como contenidos o que tipo de relaciones sintéticas son posibles entre ellos. Tampoco pregunta por el encuentro con el objeto real a través de los sentidos, en la percepción, sino que mas bien apunta a la dación en su forma mas originaria y por ende también por la conciencia, esta vez, como horizonte de posibilidad para que la dación pueda tener lugar.

Creo aquí preciso hacer expreso que la ambigüedad de sentidos en el concepto de evidencia en Husserl ya apunta, si bien de un modo incipiente - como la irrupción involuntaria de un modo de pensar aun oculto precisamente a una diferenciación propia entre verdad como adecuación y como estado de descubierto, debido a que ella ya encierra la diferencia entre conciencia sintetizadora y conciencia como horizonte del aparecer. Es por esto que se hace necesario primeramente buscar la unidad entre la dación y el estado de descubierto en un respecto, que ya, en razón de lo expuesto, no trasgreda el ámbito fenomenal. A este elemento unificador de la fenomenología se le llamara aquí horizontalidad, pues ambos proyectos comparten no solo el edificarse en torno al aparecer mismo y una acreditación adecuada a este, sino que también y por sobre todo en torno al entendimiento fundamental de que todo aparecer tiene lugar en un horizonte de posibilidad.

La diferencia fenomenológica entre Husserl y Heidegger surge del carácter de tal horizonte. Esto debe el pensamiento heideggereano al padre de la fenomenología: el aparecer y su horizonte en general, sin los cuales, la obra de Heidegger no es concebible. Heidegger de este modo no puede ser leído respecto de Husserl como una mera corrección sino que como un muy especifico desplazamiento del horizonte del aparecer.

Heidegger considera, como es sabido, que para dar cuenta del fenómeno de verdad originario, esto es, para eliminar las relaciones de correspondencia de la verdad y finalmente para poder dar paso a la dimensión fenomenal del ente, el horizonte de aparición debe ser descentrado ${ }^{12}$. Esto significa que el ente que somos, debe ser reconfigurado en una modulación distinta del sujeto, respecto de la cual el fenómeno de mundo no sea ya mas entendido como el espacio externo en el que se da el encuentro con el ente circundante, sino que como una extensión especifica

${ }^{12}$ El termino "descentrado" que aquí se utiliza responde al concepto alemán Dezentrierung, proveniente de la psicología del desarrollo, pero utilizado mas tarde en el estudio de la obra de Heidegger. 
y constitutiva de su propia esencia. A este fenómeno le llama Heidegger, siguiendo con la exposición conocida de su obra, el estar-en-el-mundo y a la modulación del ente que tiene mundo, Dasein. El mundo es en Heidegger el contexto de significación para el ente que en él se muestra, es decir su horizonte de aparición. No es necesario aquí hacer una exposición detallada de esta cuestión, sino que basta con esta somera descripción en la que se da cuenta del fenómeno de mundo en Heidegger como una horizontalidad desplazada.

Ya que en un principio hemos aseverado que aquí se pretende mostrar ambos conceptos de verdad como modos de una verdad mas amplia, es necesario dar cuenta de que la evidencia tiene y mantiene una función propia dentro del contexto inaugurado por Heidegger, y no se trata simplemente de un proto estado de descubierto.

De esto se han dado varias pistas en el transcurso de este análisis, que guardan relación al proyecto y las metas que se plantea Husserl respecto de refundar la ciencia, a través de una filosofía rigurosa. La solución aportada por Husserl funda en que la conciencia no esta determinada como un absoluto cerrado, sino que se abre al modo de la intencionalidad para dejar aparecer al objeto mismo en su dimensión fenomenal. En este sentido tiene la conciencia la función de un horizonte inmanente, a diferencia del Dasein y su mundo que se instauran como horizonte transcendente para el aparecer del ente ${ }^{13}$. El carácter inmanente que le plasma Husserl a la horizontalidad posibilita un modo de acceso concreto al ente, es decir en su darse intratemporal, lo que sustenta un modo de conocimiento - dentro de esta limitación - ilimitadamente valido. Se trata en este tipo de acceso del acotamiento del aparecer del ente al momento de su recepción en la conciencia, pudiéndose así fundar un modo de conocimiento científico y riguroso. Por otro lado el horizonte transcendente que plantea Heidegger determina fundamentalmente el acceso al ente en vistas a sus posibilidades histórico-temporales de aparición. Con esto no se pretende en absoluto negar que la historicidad del acceso transcendental al ente no conlleve su propia rigurosidad, pero no es en ningún caso, aquella del conocimiento científico refundado por Husserl. Lo que se obtiene del ajuste de estos conceptos es, a fin de cuentas, dos modos de acceso al ente siempre desde una determinación horizontal y por tanto no excluyentes: el primero de modo inmanente referido a la circunscripción del ente a una aparición especifica y acotada, que funda modos

\footnotetext{
${ }^{13} \mathrm{La}$ diferencia entre horizonte inmanente y transcendente esta pensada aquí en un contexto histórico general y no en relación al uso especifico que se le da a estos términos dentro de la obra de Husserl. El uso general de estos conceptos como se los pretende utilizar aquí no apunta a la diferencia entre real e ideal, sino que a la "ubicación" del horizonte de aparecer del ente en su dimensión fenomenal.
} 
de conocimiento técnico-concretos y el segundo como un acceso transcendente, que apunta a la posibilidad histórica del aparecer del ente en general.

De este modo es posible aseverar que la conjunción de ambos conceptos da por resultado una verdad netamente fenomenal de carácter horizontal que tiene la capacidad de fundar tanto un modo intramundano como uno mundano de accesos no excluyentes al ente.

\section{Conclusión}

En este sentido es justo aseverar que los modos de lectura que hemos presentado de los proyectos en cuestión parecen inhibir el momento de cohesión de la fenomenología y fallan en su intento de dar con un concepto de verdad amplio y adecuado a las diversas dimensiones fenomenales del ente. Es necesario poner atención especialmente a la segunda lectura descrita. Esta a diferencia de la primera parece emanar desde la fenomenología misma, guiada por una comprensión ambigua del concepto de historia por parte del propio Heidegger: Se describe el movimiento de la historia como un entramado de posibilidades que surgen y se agotan para forzar la comparecencia de otras nuevas posibilidades. Es decir las transformaciones de fenómenos históricos suceden de modo procesual. El fenómeno que irrumpe se comienza a dibujar lentamente hasta encontrar su forma concreta. Así al menos es descrita la transformación de la verdad en Platons Lehre von der Wahrheit. Curiosamente esto lo concede Heidegger en el paso de la verdad originaria a la llamada verdad metafísica ${ }^{14}$, pero se lo niega a Husserl, en el paso inverso. El presupuesto heideggereano de que la transformación que sufre la verdad hacia el estar al descubierto es tal que tiene exclusivamente lugar dentro de su propia obra, es por lo menos llamativo, ya que justamente atenta con el presupuesto fundamental de que la historia del ser se trata de un proceso descentrado, que no esta en manos del hombre. No solo respecto de la transformación de la verdad, sino que de muchos otros fenómenos fundamentales, el lector hace de testigo de una pugna en el corazón mismo de la obra de Heidegger: el pensador ora a favor, ora en contra de sus propios preceptos respecto de la historia.

\footnotetext{
${ }^{14}$ Son varios los pasajes en Platons Lehre que dan cuenta de esta dualidad, el siguiente es uno de los más claros en este respecto: "In gewisser Weise muß Platon jedoch die ,Wahrheit" noch als Charakter des Seienden festhalten, weil das Seiende als das Anwesende im Erscheinen das Sein hat und dieses die Unverborgenheit mit sich bringt. Zugleich aber verlangt sich das Fragen nach dem Unverborgenen auf das Erscheinen des Aussehens und damit auf das diesem zugeordnete Sehen und auf das Rechte und die Richtigkeit des Sehens. Deshalb liegt in Platons Lehre eine notwendige Zweideutigkeit." (p. 137).
} 
MICHELOW, D. Phenomenology, truth, and horizon. Trans/form/ação, Marília, v. 41, n. 1, p. 209-222, Jan./Mar., 2018.

ABSTRACT: This article takes up the long discussion between the two main concepts of truth in phenomenology - Husserl's concept of evidence (Evidenz) and Heidegger's concept of discoveredness (Entdecktheit). Arguments are presented against the two prevailing analytical tendencies which, following different paths, have both yielded the conclusion that there is an unbridgeable mutual exclusion between the two concepts. It is argued instead that the two concepts are complementary modes of a broader phenomenon of truth. Finally, the article critically highlights the understanding of history in Heidegger which, it is shown, had a negative impact on a unifying vision of phenomenology.

KeYworDs: Evidence, Discoveredness, Truth, History, Phenomenology.

\section{REFERENCIAS}

ADORNO, T. W. Jargon der Eigentlichkeit: zur deutschen Ideologie. Frankfurt am Main: Suhrkamp, 1970.

GADAMER, H-G. Wahrheit und Methode. Tübingen: Mohr Siebek, 2010.

HABERMAS, J. Der philosophische Diskurs der Moderne: zwölf Vorlesungen. Berlin: Suhrkanp, 1988.

HEFFERNAN, G. Bedeutung und Evidenz bei Edmund Husserl. Bonn: Bouvier Verlag Herbert Grundmann, 1983.

HEIDEGGER, M. Platons Lehre von der Wahrheit. In: Frankfurt am Main: Klostermann, 1976. p. 203-238. Wegmarken (GA 9). $2014 / 2015$.

. Überlegungen/Anmerkungen (GA 94-97). Frankfurt am Main: Klostermann, . Ser y tiempo (GA 2). Santiago: Editorial Universitaria, 2015.

HERRERA, D. Verdad y evidencia en Husserl. Ydeas y Valores, Bogotá, v. 36, n. 70, p. 33-48, 1986.

HERRMANN, F-W. v. Wahrheit - Freiheit - Geschichte: eine systematische Untersuchung zu Heideggers Schrift "Vom Wesen der Wahrheit". Frankfurt am Main.: Kolstermann, 2002.

HUSSERL, E. La idea de la fenomenología. Traducción de Manuel Gracia-Baró. México, D.F.: Fondo de Cultura Económica, 1982. . Investigaciones lógicas (HUA XVIII). Madrid: Alianza Editorial, 1999.

LUGO, E. Dos conceptos de la verdad en las investigaciones lógicas de Husserl. Anuario Filosófico, Navarra, v. 3, n. 1, p. 167-183, 1970. 
MARTEL, C. Heideggers Wahrheiten: Wahrheit, Referenz und Personalität in "Sein und Zeit”. Berlín: De Gruyter, 2008.

SALLIS, J. Heidegger und der Sinn von Wahrheit. Frankfurt. am Main.: Klostermann, 2012.

TUGENDHAT, E. Der Wahrheitsbegriff bei Husserl und Heidegger. Berlin: De Gruyter, 1970.

ZAHAVI, D. Husserls Phänomenologie. Stuttgart: UTB, 2009.

Recebido: 23/05/2016

Aceito: 14/03/2017 
MICHELOW, D. 